INTERNATIONAL BULLETIN OF BACTERIOLOGICAL NOMENCLATURE AND T AXONOMY

$\begin{array}{llll}\text { Volume } 11 & \text { No. } 1 & \text { January 15, } 1961 & \text { pp.23-28 }\end{array}$

\title{
RESOLUTION OF THE CONFERENCE ON TAXONOMY OF ACTINOMYCETES, HELD IN MOSCOW FROM JUNE 8 TO JUNE 10, 1960
}

(The following communication was received from Prof. N. A. Krassilnikov.)

"Organisation Committee of the Conference on Actinomycetes

Taxonomy. Lenin pr., 33,

Moscow, USSR.

Dear Professor Buchanan,

I am sending you the Resolution of the Conference on the Actinomycetes Taxonomy. The Conferencewas held in Moscow, 8-10 June 1960.

With best regards,

Prof. N. A. Krassilnikov

President of the Organisation

Committee."

After hearing the reports and discussion of materials presented onclassification of actinomycetes, the Conference adopted the following resolution:

1. In recent years here in the USSR as well as abroad, the research scientists are paying increasing attention to the problem of classification of the actinomycetes.

The growth of interest in this problem is primarily due to the fact that actinomycetes as producers of antibiotics, vitamins and other biologically active substances and organisms, represent one of the most important groups of microorganisms.

In connection with this there is an obvious need for prompt and exact differentiation of the actinomycetes which have acquired special practical significance.

2. In recent years the number of newly described species of actinomycetes has greatly increased.

However, the Conference regrets that some investigators when describing new species and giving names for them, as well as when they redescribe old species, fail to observe regularly the requirements of the International Code for Botanic and Microbiological Nomenclature and Taxonomy.

3. Some of the investigations devoted to the new species 
often fail to give a complete comparative description of microorganisms with reference to previous publications and sources in the scientific literature.

4. Different investigators often use non-standard and not commonly used substrata which offer other investigators no possibilities of making a comparative study of those cultures which had been described as new species.

5. A serious difficulty encountered in development of a harmonious classification reflecting the corresponding natural differentiation of microorganisms is presented by the fact that thus far among the research scientists working in the field of systematization of microorganisms and actinomycetes in particular, there is no agreement as to the importance and value of certain features and properties indetermination of species.

In view of the above mentioned facts in the classification of actinomycetes and in their nomenclature, there is still much confusion which generally obstructs progress in the study of the actinomycetes.

In consequence of the considerable general theoretical and practical importance which the classification of actinomycetes has at present the Conference has agreed that the following measures are necessary:

I. In description of new species, in re-defining those which had been described earlier and in adopting new names for them, strictly to observe The International Code of Botanical and Microbiological Nomenclature.

Fürther, when describing new species one should make reference to the relevant data published earlier elsewhere in the literature and give the results of the comparative study conducted earlier with related cultures of the type under consideration. It is obligatory to give differential diagnosis.

II. Assuming that availability of type cultures of actinomycetes would considerably facilitate the work involving classification of newly isolated cultures the Conference deems it necessary to entrust the special Committee with a study of the actinomycetes cultures available at various biological museums and select from their number those which can be recommended as standard.

The selected standard cultures should be kept in museums of live biological cultures at the following research institutions: The Institute of Microbiology of the USSR Academy of 
Science, the Institute of New Antibiotics of the USSR Academy of Medical Sciences, The All-Union Research Institute of Antibiotics (Moscow), and at the Leningrad Institute of Antibiotics.

The work on compiling the title list of standard cultures is to be entrusted to the Commissionconsisting of the following scientists:

N. A. Krassilnikov

A.I. Korenyako

O.I. Artamonova

G. F. Gause

T.B. Preobrazhenskaya

M. A. Sveshnikova

V. D. Kuznetzov

S. M. Rudaya

N.K. Solovjeva

V.A. Tsyganov
The Institute of Microbiology of the U.S.S.R. Academy of Sciences

The Institute of the USSR Academy of Medical Sciences for the Research on New Antibiotics

The All-Union Research Institute of Antibiotics

The Lening rad Institute of Antibiotics

Compiling of the list of standard cultures is to becompleted by January 1, 1961 .

III. The Conference entrusts the above mentioned Commission with the following:

a) to make detailed studies in order to redefine by isolation those cultures of actinomycetes whose allocation to definite species is still doubtful. Investigation of such cultures should be incorporated in the plans for joint studies to be conducted by the respective Institutes;

b) to resolve the various issues important for systematized classification.

IV. With the aim of eliminating differences in descripttion of cultures the Conference constitutes a special SubCommission whose members are N.I. Nikitina, A. G. Kutchaeva (The Institute of Microbiology of the USSR Academy of Science); T.S. Maksimova, E.S. Kudrina (The Institute of the USSR Academy of Medical Sciences for the Research of New Antibiotics); A. A. Melnikova (The All-Union Research Institute on Antibiotics); and K. Vinogradova (The Laboratory of Antibiotics of the Moscow State University) with: 
a) compilation of a list of media to be considered as obligatory in the study and description of new species of actinomycetes, taking into account, in compiling it, also those media which have been developed by the Soviet scientists and mentioned in the reports read at this Conference;

b) development of a scheme to be used for description of the cultural, morphologic, and other properties of actinomycetes, with special attention being paid in it to the methods to be applied for identification of each specific feature:

c) the Sub-Commission should have its work completed before January 1, 1961.

V. The Conference has decided to entrust to the Commission whose members were mentioned in the paragraph II of the present Resolution, the consideration and approval of the list of media recommended by the Sub-Commission and the scheme for description of cultures and see to the publication of these findings during 1961.

VI. The Conference deems it necessary that all the newly described cultures of actinomycetes be transferred to special Museums of live cultures at the Institute of Microbiology of the USSR Academy of Sciences, in which should be stored all the cultures of actinomycetes described in the literature.

All the cultures of any practical value and their varieties are to be kept in the live culture Museum of the All-Union Research Institute of Antibiotics.

VII. The Conference attaches great significance to the study of variability in actinomycetes as an important guide in systemization. Apart from enriching our understanding of the process of evolution, i.e. our concepts on parental relations between microorganisms and relations between separate species the study of variation provides the investigator with needed data as to possible forms of existence of cultures of certain kinds under defined conditions.

First of all a thorough study should be made of the variability of those cultures which are extensively used for practical purposes, as wellas of the most important representatives of the type cultures.

Special attention should be paid to the study of possibilities of changing such important properties used for determination of species such as the ability to produce certain antibiotics and pigments; also very important is the study of structure of spores, of cells, etc.

The Conference considers it to be expedient to continue 
further research on hybridization of actinomycetes with the purpose of collecting data on parental relations among various groups of actinomycetes.

VIII. The facts collected on the use of actinophages in classification of actinomycetes clearly indicate that indications of susceptibility or stability in biological cultures to certain specific actinophages can be advantageously applied for differentiating them in some cases into species, and in others-into types within a certain species.

However the number of specific actinophages available at present is rather limited.

The Conference considers as an important task the further extension of investigations in the field of isolation, study and extensive practical verification of the specific qualities of actinophages. The Conference applies to the Institute of Microbiology of the USSR Academy of Sciences with the suggestion to gather a collection of typical actinophages and to make available to other research institutions.

At the same time the Conference attaches a considerable significance to the thorough study of lysogeny phenomena and the effect of symbiotic actinophages upon the specific biological properties of lysogenic cultures.

The Conference attaches significance to the extension of investigations on the application of serologic methods for classification of actinomycetes.

In connection with such serologic studies the following measures are necessary:

a) perfection of methods for obtaining the highly active specific antigens and serums as well as perfection of methods for investigation of serologic properties of the cell and its separate parts;

b) study of serological properties of experimentally derived varieties of type cultures;

c) to approach the All-Union Institute of Antibiotics with the request that it make available for the purposes of research serums which are specific for the type cultures of actinomycetes.

IX. The Conference also attaches importance to the following measures:

a) extending investigations in the field of studying species peculiarities in study of microscopic morphology of actinomycete colonies and of observing their specific features in microscopic sections; 
b) generalization of the collected cytological data on development of microscopic morphology of the mycelium of industrial cultures as found in extensive fermentation;

c) sponsoring special investigations on study of cytochemical and biochemical properties of actinomycetes with the purpose of assessing the possibilities of making use of these properties for their differentiation;

d) to considerably extend all kinds of investigations of pigments.

$X$. The Conference deems it necessary to address the USSR Taxonomy Committee proposing establishment of a special Sub-Committee under it on taxonomy and systematization of actinomyces, and to transmit its membership to the International Committee on Taxonomy of Microorganisms.

To instruct such Sub-Committee to publish either in the magazine "Microbiologiya" or "Antibiotiki", or to publish as a separate booklet the International Rules of Taxonomy and systematization of microorganisms (Code of Nomenclature of the Bacteria and Viruses.)

To make the appropriate arrangements for publishing the Code of Colours. Pending its publication the Conference recommends to all the use of the Bondartsev Code.

XI. The Conference attaches special importance to publication of instructions on systematization of actinomycetes as well as instructions on methods for their investigation.

XII. The Conference addresses to the All-Union Research Institute of Antibiotics a request to supply various research institutions with cultures immunable to certain antibiotics.

XIII. The Conference deems it of particular importance that various research institutions working in the field of systematization of actinomycetes be provided with " standard" antibiotics. 